N r6. Extended Gaseous Nebul In Cygnus. Photographed by Prof. E. E. Barnard with the Bruce Telescope.

These two wonderful filamentary nebulæ are parts of a still larger ring, the fainter portions of which can be photographed, but probably have never been seen with the eye. They are sometimes called the " cirrus nebula " because of their resemblance to the cirrus clouds frequently seen in the sky. They are gaseous, composed of nebulium, hydrogen, and helium. They are of enormous size, each of the two brighter nebulæ being over a degree in length, and even the slenderest filaments must be hundreds of millions of miles in width.

It will be noticed that the nebula at the left of the slide lies along the line of separation of a region thick with stars from a region noticeably less dense in stars. It has not yet been possible to detect any motion of these filaments with respect to the stars, but probably it will ultimately be measured when photographs are available separated by a long period of years.

During this long exposure of nearly six hours, a meteor flashed across this part of the sky, and its impression was caught on the plate. It is brighter than some of the filaments of the nebulæ, but there could be no greater contrast in actual size or brightness, for the meteor was only a small pebble, or perhaps a grain of sand, burning itself out by friction in our atmosphere, and probably less than roo miles away, while the nebula is several hundred light-years distant from us.

N 5 I. Planetary Nebula in Gemini, N.G.C. 2392. Photographed by Prof. E. E. Barnard.

Planetary nebulæ, of which there are only about I50 catalogued, are all apparently small in size, are rather dense, and in general have well-defined outlines resembling ellipsoidal shells, with a Wolf-Rayet star at the centre. They are uniformly gaseous, their inner parts containing helium, nebulium, and hydrogen; their outer parts, hydrogen only.
Since they have a tendency to lie in the plane of the Milky Way, they probably belong to our own system. One planetary nebula has been found to be distant I 40 light-years, but the average distance is probably about Iooo light-years. From rather uncertain data some are found to be rotating about an axis with periods of from 600 to 14,000 years, their masses being three to one hundred times that of the sun, but with densities of about one-millionth that of our atmosphere.

The two photographs are of the same nebula, but taken with the 25-foot and the Ioo-foot focus, respectively, of the 6o-inch reflector at Mount Wilson Observatory. The first is over-exposed in the centre in order to bring out the details of the faint outer ring, while the one of large scale shows the inner structure. The appearance of this nebula is unique, and bears little resemblance to that of other nebulæ.

The fact that planetary nebulæ have an average radial velocity of 24 miles per second discountenances the theory that they condense into the early types of stars, $\mathrm{O}$ and $\mathrm{B}$, whose velocities are markedly less. The radial velocity of this nebula is 50 miles per second. It has been suggested that planetary nebulæ are the wrecks of ancient collisions among stars.

We hope that the effort which the publishers of these illustrations and descriptions are making to spread the knowledge of astronomy in so interesting a manner will meet with the success which it deserves. It will be understood that the work of distributing lantern slides and photographs, or descriptions of them, is not a matter of business with the Yerkes Observatory, as the proceeds from sales cannot do more than cover the cost of manufacture, quite apart from the time spent by scientific members of the staff of the Observatory in selecting the subjects, supervising the photographic work, and writing the descriptions.

Copies of an illustrated catalogue of the photographs made at the Yerkes Observatory, or of a selected list of one hundred slides, may be obtained from the University of Chicago Press, Chicago, Illinois, U.S.A.

\title{
Brain and Speech.
}

\section{By Tudor Jones, M.B.}

$A \mathrm{~S}$ the conclusions which Dr. Henry Head bases upon his extensive researches into the phenomena of aphasia are now approaching a comprehensive form, ${ }^{1}$ the present is a convenient time to attempt a summary of his views. Fourteen years ago, in the light of his work on the part played by the brain cortex in sensation, it became clear that new tests must be devised before the clinical investigation of aphasia could produce any satisfactory result.

It is less necessary to describe here the elaborate series of tests which Dr. Head invented, every one of them new or used in a new way, than to direct attention to the principles which guided him in this important part of his work. ${ }^{2}$ Foremost among these was the conception, which a study of his own work and that of Sherrington and his collaborators led Head to formulate, that cortical injury, so far from removing a strictly definable anatomical "centre," disturbs a highly organised act, the very variable end-point of a whole march of events occurring in the central nervous

' "Speech and Cerebral Localisation," by Henry Head, Brain, vol. xivi. Part iv., r923 (London: Macmillan and Co., Ltd.) 6s. net.

${ }^{2} \mathrm{~A}$ description of the tests appeared in Brain, vol. xliii., 1920. system. The effects of a destructive lesion of the brain cortex are all negative. They do not reveal the primitive elements out of which speech issues, nor do they break it up in accordance with our conceptual terms in describing it. What they do is to prevent, in part, the activity of a highly organised group of functions all at the cortical level. Head insists very strongly upon the negative characters of this interruption, distinguishing them from such positive effects as the spasticity of hemiplegia, which reveals the uncontrolled activity of neurons at a different level. Thus his first aim was to determine with the greatest possible accuracy what it was that the patient could not do, or could do only sometimes or with difficulty. The tests at length devised are not considered to be complete or incapable of improvement; but that they constitute in themselves a great advance in clinical method has been admitted even by those who, for the present, are unable to adopt Head's more theoretical conclusions.

This great gain is, however, less than that which must proceed from due observance of the principle itself: that what we must strive to ascertain is not the defect 
in such conceptual terms as may occur to us or be forced upon us by an imperfect and misleading psychology ; but the defect stated in cortical terms, in terms of the special function actually removed from the great chain of functions of which speech in all its forms is the last link. The terms "motor," "sensory," "emissive," "receptive" are conceptual no less than " apraxia," "agraphia," and all the train of Greek words which merely translate the plain English for various gross disabilities. Not in our terms, which describe only finished products of cortical activity, but in terms of purely cortical function, what absence of one or several acts leading to the formulation and expression of symbols can be discovered?

This is surely one of those rare questions, which have a certain power of illumination of their own apart from any answer which may be found for them. The complete answer is for the present an ideal involving an account of the innumerable integrations of the central nervous system and of their modes of action. Yet in defining so vast a problem, Dr. Head has concurrently swept aside the crude theories of the past half-century in regard to aphasia, and has carried us far towards a rational understanding of the functions of speech. ${ }^{3}$

After the outbreak of the War, Head was able to apply his tests to a class of patient vastly different from the aged aphasics of civilian practice with their arterial degeneration, diminished intellectual capacity and mental depression. Many "were extremely intelligent, willing and anxious to be examined thoroughly. As their wounds healed they were encouraged and cheered by the obvious improvement in their condition. They were euphoric rather than depressed." Moreover, while many vascular lesions tend to destroy the substance of the brain, the damage caused by gunshot wounds often tends to be most extensive superficially. One only of the cases upon which the research is based was a civilian, an intelligent woman, from whose brain an extra-cerebral tumour was removed by operation.

When Pierre Marie first attacked the "classical" presentation of the facts of aphasia, he attributed the "sensory aphasia" of Wernicke to a defect of general intelligence and of special intelligence of language. Head's first finding contradicts this view. A man who in general conversation is unable to express his thoughts or comprehend the full significance of words and phrases appears more stupid than others. His isolation from the sources of mental life diminishes his field of thought, and thus many aphasics gradually deteriorate. But "general intelligence" (the existence of which Hughlings Jackson denied) is not primarily affected. Behaviour suffers specifically. What cannot be done in one way can be done in another. "All my patients could choose some familiar object, colour, or geometrical figure, which corresponded to the one shown to them." No symbolic formulation intervenes necessarily between perception and such an act of choice. When a unilateral lesion of the brain disturbs the use of language, any act of mental expression which demands symbolic formulation tends to be defective, and the higher its ${ }^{3}$ The " classical" localisation of cortical function was completed with
Wernicke's localisation of a centre for " auditory images" in 1874 . Despite
Hughlings Jackson, the subject then entered upon a phase of complete
sterility which lasted until Marie's " Revision of the Question of Aphasia"
in rgo6.

NO. 2840, VOL. I I 3$]$ propositional value the greater difficulty will it present. Any modification of the task which lessens the necessity for symbolic representation will render its performance easier.

It is remarkable how definitely Head's observations cut up the classical terminology and unmask its latent assumptions. Many patients who cannot name objects can choose them to oral as well as printed command. "Once the word has been presented to the patient, it calls up an image which corresponds to some object before him "; but he cannot himself reproduce the appropriate symbol on demand. The more abstract the symbol the greater the difficulty which its invocation affords. Colours are more difficult to name than objects-i.e. crude colours, not their varied shades. A patient failed to recall the name for " black," but was perfectly at home with the more concrete "what you do for the dead," and "dead," to which he shortened this phrase, served perfectly as a more accessible synonym. Moreover, we make use of many subtle symbolisms (how many?) besides those indicated on the conventional brain charts. "The short and long hands of the clock have acquired a significance which converts each of them into a direct symbol, and they are confused or wrongly used in many forms of aphasia." Galton's classical article described many highly individualised uses of such symbols. Patients who cannot make use of the conventional time nomenclature, either in words or by the peculiar symbolism of the clock itself, will describe events more concretely as "when we went there"; they recognise the passage of time intervals and are punctual for appointments.

Again, not only have sensory impressions to be " formulated" in terms of a symbolism which employs other forms of sensation besides hearing and vision, the whole forming an aptitude acquired laboriously in the lifetime of the individual, but also the relations between sensory impressions must be expressed in accordance with the same conventions. As Elliot Smith, approaching the question from morphology, has pointed out, man's immediate ancestors possessed high powers of discrimination before man developed speech and invented his symbolisms to register differences (relations) apparent to him Like speech, this is a cortical function but earlier acquired. "A patient with his eyes closed may be able to point to the position of various objects in some familiar room with regard to himself, although he cannot formulate their relation to one another. He can say "The window is there, the fireplace there," but is unable to express the situation of the one with regard to the other. This makes it impossible to draw a ground plan, which demands somewhat complicated acts of symbolic formulation. Similarly illuminating disabilities arise among aphasics in their power to handle money with its complex symbolism. The primary significance of words, figures, and coins may be appreciated, but not their deeper significance in relation to one another. The processes of arithmetic have to be constantly reformulated in order that they may be correctly carried out.

It will be clear that the disorders of behaviour observed by Head involve a wide range of acts from mechanical acts up to exercises in formal logic; they manifest themselves most profoundly in the use of 
symbols, i.e. words, numbers, and analogous conyentions. "The processes which underlie an act of speech run through the nervous system like a prairie fire from bush to bush ; remove all inflammable material at any one point and the fire stops." Clearly, it is as reasonable to call the site at which there is discontinuity of the nervous mechanism a "speech centre " as it would be to call the place where the fire ceased a " fire centre." Most so-called "centres" in the nervous system are nodal foci, where central neural activities undergo integration and other changes in relation to one another. But the evidence laboriously collected by Head displaces from the classical "centres" all that it has been customary to regard as resident within them.

These anatomical sites are themselves not functional parts from which words or other acts spring ready formed; " there are no 'centres' for speaking, reading, writing, or other forms of behaviour comprised in the normal use of language." There are merely situations where the normal stream of neural activity may be interrupted with results which may or may not be characteristic. The view that there can be anything characteristic, in our purely linguistic terms, is set aside as an absurdity. We must learn to speak the language of the cortex itself before we can state its disorders in appropriate terms. Acts of symbolic formulation and expression " are built up on a. level of integration superior to that of motion, and are of a higher order than sight or hearing." Consequently, the clinical manifestations cannot be classified as "motor," "visual," or " auditory" defects of speech. Head has given a death-blow to the classical theory of aphasia and has thrown the whole conception of "centres" into the melting-pot, for even motor "centres" in the cortex are not primary foci from which movements are initiated and determined, but rather "the means by which we are enabled to interfere with otherwise automatic acts."

We must now inquire what substitutes can be found for the "linguistic" terminology. The disorders of speech following cortical injury reveal themselves to us as disabilities of various kinds, associated with symbolic formulation and expression. The analysis of these disabilities made by the "classical " technique meant nothing. Do the more discriminating methods of Head bring us nearer to what I have ventured to call the language of the cortex itself? I have used this phrase because Head's argument does seem to me to discriminate between the disturbances of cortical function and the disturbances of speech which alone are subject to clinical investigation. It is the whole burden of his insistence that the diverse defects of speech do not reveal the elements out of which the use of language is built up. These elements are functions of the central nervous system. Neurologically, what is all-important is that we should move towards some understanding of these elements. Clinically, we are justified in grouping the manifestations of interrupted function in whatever way the facts determine, and Head has effected such a grouping himself, being able, further, to show a corresponding anatomical localisation. But the clinical and neurological demands must not be confused. It is to ward off such confusion that Head has taken up an attitude towards localisation that has mystified many of his opponents. He has given names to his groups (verbal, syntactical, semantic and nominal), and has assigned each group to an area of the cortex more or less definite. ${ }^{4}$ Indeed, the facts appear to justify a greater definiteness than he is willing to claim. But he would have preferred to identify his groups by some purely conventional sign. Clinically the groups are clear cut.

There should be little difficulty in classifying an aphasic in accordance with Head's system without very minute investigation. There is something distinctive about each group, and the distinguishing characteristics are readily revealed by the application of Head's system of testing. But, if our work ended there, we should have only added a new (albeit a better) clinical convention to the study of aphasia. We should be no nearer to the elucidation of cortical function in regard to speech. The verbal aphasic has difficulty with verbal forms of expression. The structure of his words is abnormal. They may be so badly pronounced as to be scarcely recognisable; but they are applied correctly. The significance of words is retained, and the patient can appreciate jokes presented to him in pictures. He can play card games and draw plans. The syntactic has plenty of words but their production is ataxic. Comprehension of the meaning of words is always in excess of their use. The arhythmical character of spoken words extends to phrases, so that phrasal memory is defective, and conversation difficult or impossible. The order of terms in a simple phrase is often reversed, and pictures are "read " from right to left. Nominal defects show themselves as an inability to name things, which becomes more marked the more abstract the nature of the things. Relations cannot be identified by appropriate word labels. The patient fails to choose correctly to oral commands, and cannot draw a ground plan, although he knows where things are. He cannot play card games because they demand rapid and correct recognition of names and an ability to register a score ; but chess, draughts, and dominoes may be played correctly. The semantic fails to recognise the full significance of words and phrases, apart from their verbal meaning. The "point" of a picture escapes him. He cannot play games or put picture-puzzles together. He cannot formulate a general conception of what he has been told or has read for himself. But he does not forget people or places ; he can recall spontaneously events both recent and remote.

It is Head's thesis that there is nothing exclusive about the acts which are disordered by cortical injury. They imply highly integrated functional arrangements, developed originally for other simpler purposes. But with increasing aptitude the functional adaptations on which the use of language relies become engulfed in the automatic activities of the central nervous system. $\mathrm{He}$ has suggested a differential but nevertheless serial classification of functions involved in the simplest acts of speech, and in so doing has opened up the way for further investigation of a very promising kind. Neurology as well as clinical medicine, but above all psychology, is indebted to him for a great pioneer work.

4 The localisation of cortical injuries in Head's cases was as follows : Verbal defects, the lower portion of the central convolutions and the parts beneath them; syntactical, an area above and below the superior temporal fissure; nominal and scmantic, between the post central fissure and the occipital region, the latter the more postero-medial.

NO. 284 O, VOL. I I 3$]$ 